\title{
Practitioner compression force variation in mammography: a 6 year study
}

\section{Introduction}

In mammographic practice, breasts are compressed until adequate thickness reduction is induced. Various descriptors have been proposed to indicate when enough compression force has been applied ${ }^{[1-5]}$. The main aims of compression include the requirement to improve image quality ${ }^{[6]}$ and the need minimise breast radiation dose

${ }^{[7]}$. However, within the National Health Service Breast Screening Programme (NHSBSP), there are no specific guidelines for optimal compression force levels required to achieve effective breast thickness reduction, other than a statement indicating that 'the force of the compression on the x-ray machine should not exceed 200 Newtons ${ }^{[8]}$.

Previous research ${ }^{[9]}$ has established that practitioners vary in the amount of compression force they apply to breast tissue during mammography. This finding was independent of specific client characteristics (e.g. breast density). This research involved the cross sectional evaluation of 14 practitioners and 344 clients' compression force data on one mammography unit. Statistical analysis demonstrated a highly significant difference in mean compression used by different practitioners ( $\mathrm{p}<0.0001$ for each BI-RADS density). Practitioners applied compression force in using low, intermediate or high compression force, with no significant difference in mean compression force within each group $(\mathrm{p}=0.99, \mathrm{p}=0.70, \mathrm{p}=0.54$, respectively). It concluded that practitioners routinely apply either low, intermediate or high levels of compression force. Consequently, it was suggested that the amount of compression force applied to the breast could be highly dependent upon the practitioner.

As NHSBSP requires serial imaging to occur at regular intervals, with images reviewed to assess for subtle changes ${ }^{[10]}$, if compression force variability between practitioners exists then comparison between images over time may become more challenging. Additionally, and importantly, client experience may vary too and this may affect re-attendance rates. As such, we conducted a retrospective analysis to establish whether practitioner variations in compression force existed over time. For this analysis we identified a consecutive analogue sample from NHSBSP client data over 3 screening cycles -6 years in total. 


\section{Materials / Method}

\subsection{Study Characteristics}

The study was performed in a regional breast screening service located in the North of England (UK). Hospital audit and University ethics committees approved access to a sample of 500 clients from which data could be drawn. In order to reduce variability between mammogram machines, data was gathered from one static site, using one mammogram machine (analogue GE DMR+ mammography machine; Chalfont St. Giles, UK). The machine was operating within NHSBSP and manufacturer specifications ${ }^{[11-12]}$ during the study period.

\subsection{Client Sample}

Analogue mammogram images and associated data were gathered retrospectively. Data was gathered from clients who attended three consecutive screens. Only three screening rounds could be included as the required data for this study was unavailable prior to 2004. Data and images were therefore included from 2004, enabling 2004, 2007 and 2010 screening rounds for inclusion.

Identification of clients who were included into this study was through a consecutive convenience sampling basis. To be included each client had to have 3 consecutive screening mammograms 2004, 2007 and 2010; their first recorded mammogram experience at 2004. Each would have had the 4 standard projections acquired (left / right CC (cranial-caudal) and left / right MLO (medio-lateral oblique). For each client the following information was recorded - size of film, breast compression force value in deca-Newtons (daN), compressed breast thickness $(\mathrm{mm})$ and the name of practitioner who performed the mammogram. The latter was coded for anonymity purposes.

Mean glandular dose (MGD) estimations ${ }^{[12]}$ were calculated retrospectively for specific clients. Together with this, breast density was established by one reader for each image using the 4 point $\mathrm{BI}-\mathrm{RADS}_{\circledast}$ scale $($ Breast Imaging Reporting and Data System $\left.{ }^{[13]}\right)-$ BI-RADS $_{\circledast} 1<25 \%$ dense, BI-RADS ${ }_{\circledast}$ 2: $25 \%-50 \%$ dense, BIRADS $_{\circledast} 3: 51 \%-75 \%$ dense, and BI-RADS ${ }_{\circledast} 4>75 \%$ dense. This reader was an experienced breast practitioner who had good BI-RADS ${ }_{\circledast}$ classification scoring agreement with 3 other experienced breast clinicians (Kappa $0.83,0.92,0.83)^{[9]}$. 


\subsection{Exclusion Criteria}

Exclusion criteria included: the inability of clients to tolerate compression force, clients who had breast pain, previous breast surgery, breast implants or cysts/abscesses, disabled clients, clients with arm/shoulder movement limitations. As the study was retrospective some client data we would have liked to consider was not available - for example point in menstrual cycle and whether the client had pain upon pressure application. Consequently these parameters could not be considered in our analysis. Due to exclusion criteria 156 of the 500 clients were not included; 344 clients remained. This represented 1032 'mammogram sets' over the 3 screening rounds - 4128 individual images.

\subsection{Practitioners}

The clients were imaged by 14 trained practitioners; these consisted of all the staff that rotated through the breast imaging department at the time of the study. They comprised of Advanced Practitioners, mammographers and Assistant Practitioners with experience ranging from 1-12 years. These practitioners were the same as those used in a previous study ${ }^{[9]}$; this permitted direct comparison of results between these two studies. The average number of mammograms performed per practitioner was 73 (range 10-146).

\section{Results}

For the 344 clients the following analysis was carried out.

\subsection{Breast Density Change}

Data was categorised into BI-RADS ${ }_{\circledast}$ breast density distribution for each mammogram visit. Only $7 \%$ of clients $(\mathrm{n}=24)$ showed a change in $\mathrm{BI}-\mathrm{RADS}_{\circledast}$ density over time. This represented a reduction of one BI-RADS $\circledast$ density grade. These clients were not removed from the sample prior to analysis in the first instance as images were analysed separately. It was only when sequential patient images were considered together that these BI$\mathrm{RADS}_{\circledast}$ density variations were removed.

\subsection{Compression Force Values}

Regardless of BI-RADS ${ }_{\circledast}$ density grade, practitioner data was first analysed for mean compression force on each mammogram projection (MLO and CC). All mammograms were assigned to the practitioner who performed the mammogram, regardless of year imaged. Figures $1 \mathrm{a}$ and $1 \mathrm{~b}$ demonstrate the mean compression force values, standard deviations and confidence intervals for each practitioner. 
Within a previous study ${ }^{[9]}$ these practitioners were placed into compression force groups because of their similar compression force means; this provided a way of classifying them. For the current study the same practitioner groupings / classifications were applied - the practitioners had similar compression force means as the previous study ${ }^{[9]}$ (rank sum correlation coefficient $=0.9$ ). The coefficient of 0.9 indicates that the practitioners performed very similarly in their compression force behaviours for both client datasets. In the current study 4 practitioners fell into the low compression force group, 7 into the intermediate group and 3 into the high group. Dispersal of practitioner grade and length of experience across the three compression groups appeared to demonstrate no particular trend for the purposes of this study.

For the low compression force practitioner group: in the MLO projection, practitioners imaged with compression force mean values (regardless of BI-RADS density grade) between 7.17 and $7.4 \mathrm{daN}$ and in the CC projection between 6 and $6.27 \mathrm{daN}$.

For the intermediate compression force practitioner group: in the MLO projection practitioners imaged with compression force mean values (regardless of BI-RADS density grade) between 8.6 and 9.6daN and in the CC projection between 7.95 and $8.71 \mathrm{daN}$.

For the high compression force practitioner group: in the MLO projection practitioners imaged with compression force mean values (regardless of BI-RADS density grade) between 12.6 and 14.daN and in the CC projection between 11.45 and $11.7 \mathrm{daN}$.

There is a highly significant difference in the mean compression force values between the practitioners in the low and the intermediate group, the low and the high group and the intermediate and the high group $(\mathrm{p}<0.0001)$; this holds true within each BI-RADS density classification.

\subsection{Breast thickness values}

Mean thickness of breast tissue for each practitioner is presented, distributed by BI-RADS ${ }_{\circledast}$ density grade, in Figure 2. There is a highly significant difference between the breast thicknesses from the intermediate practitioner group and the high practitioner group $(\mathrm{p}<0.0001)$. There is a significant difference between the breast thicknesses from the low practitioner group and the high practitioner group $(\mathrm{p}<0.001)$. There is no 
statistical difference between the breast thicknesses from the low practitioner group and the intermediate practitioner group.

\subsection{Longitudinal Assessment of Compression Force and Thickness due to Practitioner Variation}

In order to assess if there was variation of compression force and breast thickness over the three screening rounds, specifically due to practitioner variation, we applied additional inclusion / exclusion criteria. From the remaining 344 clients we assessed which clients had been imaged either: sequentially by the same practitioner for each of the 3 screens, sequentially by practitioners from the same practitioner group for each of the 3 screens, or sequentially from practitioners from different compression force groups for each of the 3 screens. From the remaining 344 clients, 134 remained for further analysis for the exacting purposes of analysing longitudinal variation of compression force and thickness.

To achieve assessment of compression force and thickness variations within these clients, we set a 'reference value' of zero to the client's initial mammogram. Any increase or decrease from that value was represented by a plus (an increase in compression force or thickness) or a minus (a decrease in compression force or thickness). The term 'maximum absolute compression force variation' is the maximum compression force difference displayed between each screening mammogram for the three years for clients. Similarly the term 'maximum absolute thickness variation' is the maximum breast thickness difference displayed between each screening mammogram for the three years for clients; their first mammogram experience (incident round) being assigned as their reference value.

\subsubsection{Clients Imaged sequentially by practitioners from the Same Practitioner Compression Force Group}

From the 134 clients, 81 were imaged by a practitioner from the same compression force group on each attendance. Of these, 6 clients had a change in BI-RADS ${ }_{\circledast}$ density over time and at this stage of the analysis they were removed to minimise any variation in compression force / thickness which may be caused by density change.

Seven clients were imaged by practitioners in the low compression force group each time they attended their 3 screens. These clients experienced maximum absolute compression force variations between the sequential screens of $-2 \mathrm{daN}$ and $+1 \mathrm{daN}$ (MLO projections) and $-2 \mathrm{daN}$ and $+1 \mathrm{daN}$ (CC projections). There were no 
statistically significant differences in the compression force values of these clients over their three screening episodes. Maximum absolute breast thickness variations between the sequential screens of these clients were $18 \mathrm{~mm}$ and $+9 \mathrm{~mm}$ (MLO projection) and $-17 \mathrm{~mm}$ and $+6 \mathrm{~mm}$ (CC projection). Again there were no statistically significant differences in the breast thickness values of these clients over their three screening episodes.

Sixty eight clients were imaged by practitioners from the intermediate compression force group each time they attended. These clients experienced maximum absolute compression force variations between the sequential screens of $-4 \mathrm{daN}$ and $+2 \mathrm{daN}$ (MLO projection) and $-3 \mathrm{daN}$ and $+2 \mathrm{daN}$ (CC projection). There were no statistically significant differences in the compression force values of these clients over their three screening episodes. Maximum absolute breast thickness variations between the sequential screens of these clients were $22 \mathrm{~mm}$ and $+10 \mathrm{~mm}$ (MLO projection) and $-14 \mathrm{~mm}+15 \mathrm{~mm}$ (CC projection). Again there were no statistically significant differences in the breast thickness values of these clients over their three screening episodes.

Over the three sequential screening rounds 14 clients were imaged by the same practitioner on each attendance. These clients experienced maximum absolute compression force variations between the 3 screens of $-2 \mathrm{daN}$ and $+2 \mathrm{daN}$ (MLO projection) and $-2 \mathrm{daN}$ and $+1 \mathrm{daN}$ (CC projection). There were no statistically significant differences in the compression force values of these clients over their three screening episodes. Maximum absolute breast thickness variations between the 3 screens of these clients were $+16 \mathrm{~mm}$ and $-15 \mathrm{~mm}$ (MLO projection) and $+17 \mathrm{~mm}$ and $-6 \mathrm{~mm}$ (CC projection). Again, there were no statistically significant differences in the breast thickness values of these clients over their three screening episodes.

In summary the clients who saw the same practitioner or practitioners from the same practitioner compression force group on their three sequential screening mammograms had no significant differences in their breast thickness or their breast compression force levels.

\subsubsection{Clients Imaged Sequentially by Practitioners from Different Compression Force Groups}

Thirty nine clients were imaged by a practitioner from each compression force group (low, intermediate and high) during their three screens in a variety of orders. As above their first screening attendance was assigned a 'zero' and changes calculated from this figure. 
These clients experienced maximum absolute compression force variations over the three sequential screens of $2 \mathrm{daN}$ and $+10 \mathrm{daN}(\mathrm{MLO}$ projection) and $+3 \mathrm{daN}$ and $+14 \mathrm{daN}(\mathrm{CC}$ projection). For these 39 clients, in order to represent this change in breast compression force longitudinally over the 3 screens, the results have been displayed time independently and averaged for the two MLO and CC projections for each attendance (Figure 3). T-Tests indicate highly significant differences in compression force values $(\mathrm{p}<0.0001)$ for CC and the MLO projections. This level of significance is the same for the low and high compression force groups, the intermediate and high compression force groups and the low and intermediate groups.

For the 39 clients, in order to represent change in breast thickness longitudinally over three sequential screens, results have been displayed time independently and include both MLO and CC projections for each attendance (Figure 4). Absolute thickness reductions between the low and intermediate group reduced by $1 \mathrm{~mm}$ (MLO projection) and $1.7 \mathrm{~mm}$ (CC projection). Absolute thickness reductions between the intermediate and high group reduced by $5.7 \mathrm{~mm}$ (MLO projection) and $6 \mathrm{~mm}$ (CC projection). Absolute thickness reductions between the low and high group reduced by $6.2 \mathrm{~mm}$ (MLO projection) and $7.7 \mathrm{~mm}$ (CC projection). T-Tests indicate highly significant differences in breast thickness reductions $(\mathrm{p}<0.0001)$ between the low and high compression force groups and the intermediate and high compression force groups for both projections. The differences between low and intermediate groups did not achieve the same level of significance; for the MLO projections there was no significant difference, for the CC projections it was significant $(\mathrm{p}<0.05)$.

MGD for the 39 clients was calculated retrospectively. These are illustrated in Figure 5. Maximum dose differences were $2.64 \mathrm{mGy}(\mathrm{MLO})$ and $1.12 \mathrm{mGy}(\mathrm{CC})$ when clients were imaged by a practitioner from a low practitioner group and then a high practitioner group. Some clients experienced differences in dose of $1.57 \mathrm{mGy}$ (MLO) and $1 \mathrm{mGy}(\mathrm{CC})$ when they were imaged by a practitioner from a low compression force group followed by a practitioner from an intermediate compression force group.

Overall percentage dose differences demonstrated a mean difference of 10.2\% (MLO) and 6.9\% (CC) when clients were imaged by practitioners from a low practitioner group followed by practitioners from a high compression force group. These dose differences would likely represent a clinically important difference and are due to the differences in breast thickness levels on mammogram acquisition (Figure 5). T-tests highlight significant dose differences between the low to high compression force groups in both projections $(\mathrm{p}<0.01)$ 
Differences from low to intermediate groups are not significant for the MLO though significant for the CC view $(\mathrm{p}<0.05)$. For the intermediate to high group for both projections there were no significant differences.

In summary, clients who saw practitioners from a different compression force group on each attendance had significant differences in their compression force levels and some of their thickness levels. Depending upon which practitioner compression force group is considered significant differences in dose have also been demonstrated.

\section{Discussion}

\subsection{Implications for practice}

Our study establishes that the amount of breast compression force seems highly dependent upon practitioner rather than client. This has implications for radiation dose and image quality consistency for sequential screening within the National Health Service Breast Screening Programme together with in the symptomatic setting.

We have highlighted four areas for consideration. Firstly, the practitioners fall into the same compression force groups as with Mercers' previous study ${ }^{[9]}$. Secondly, from a client perspective, the compression force that is applied to client breasts during each mammogram can vary over time and this is dependent upon the practitioner who images them. Thirdly, for the clients who were imaged with a practitioner from a different group on each attendance, breast compression force values are significantly different. Breast thickness reduction was also significantly different apart from between the low and intermediate compression force groups on the MLO view. This suggests that there is significance to the application of higher compression force in the reduction of breast thickness. Finally, it has been highlighted that for certain cases, the larger thickness reductions have resulted in lower mean glandular doses (MGDs). Though t tests show that some of these were not statistically significant in some cases, there has to be consideration of the clinical importance of this - doses should be kept as low as practical.

It appears that each practitioner is consistent over time in the amount of compression force that they apply. We have also indicated that there is a close correlation between mean compression force values from this study and Mercer's cross sectional study ${ }^{[9]}$. This suggests that individual practitioners are applying compression force 
consistently over time, and also within different client groups. This could mean that practitioners are applying their own tolerance levels to compression force application. We have also demonstrated that changes in BI$\mathrm{RADS}_{\circledast}$ density grades made little difference to the practitioner's behaviour in their application of compression force. This again could suggest that practitioners are applying compression force to the breast using their own tolerance levels regardless of breast type.

The relevance to clients being imaged by practitioners applying different levels of compression force may give rise to different levels of pain and discomfort experienced whilst having mammography and this may have consequences for future attendance. Studies ${ }^{[14-18]}$ have suggested varying thresholds of compression force for pain tolerances varying from $9 \mathrm{daN}$ through to $16 \mathrm{daN}$. As such, consistency of optimal compression force applied over time could be paramount in the maintenance of client experience. The same argument would hold true for consistency of image quality over time.

Our data has demonstrated statistically significant variations in breast compression force and breast thickness levels when clients are imaged by different practitioners over their 3 screening rounds. Our study has also demonstrated that clients imaged by the same practitioner on each screen have less breast compression force and breast thickness variation. It is likely that these clients have had more a consistent experience.

For the third and final issues, breasts might be imaged with breast thickness reduction (rather than compression force) in mind in order to reduce radiation burden ${ }^{[1,4]}$. This will likely achieve better consistency of breast dose and image quality ${ }^{[19]}$ for clients imaged serially within the NHSBSP.

\subsection{Limitations}

Our study has several limitations. Firstly, this study was at a single site with a relatively small group of mammographers. The study has now been extended to a multicentre study in order to assess if the results will be similar at other screening centres. Secondly, as this was retrospective important factors such as point in menstrual cycle, breast pain upon compression force and weight changes, for example, could have effect on the results of this study. 


\section{Conclusion}

We have established that compression force and breast thicknesses can fluctuate for the same client when they are imaged by different practitioners. Implications from this can result in variations in mean breast glandular dose between 3 yearly screening events. The possibility exists for variations to occur in image quality and lesion visibility. Given that compression force differences can occur over time it is possible that client experience may vary too with possible implications to clients screening attendance within the future. 


\section{REFERENCES}

1. Long S. The handbook of mammography, $4^{\text {th }}$ edn. Edmonton: Mammography Consulting Services Ltd, 2000 .

2. Wentz G. Mammography for radiologic technologist. New York: Mc Graw Hill, 1992.

3. Kopans D. Breast Imaging, $3^{\text {rd }}$ edn. Lippincott: Williams and Wilkins, 2007.

4. Eklund GW, Cardenosa G. The art of mammographic positioning. Breast Imaging: Current Status and Future Directions. Radiologic Clinics of North America. 1992; 30:21-53.

5. Poulos A, McLean D. The application of breast compression in mammography: a new perspective. Radiography. 2004; 10:131-137.

6. Poulos A, McLean D, Rickard M, Heard R. Breast compression in mammography: How much is enough? Australias Radiol 2003: 47:121-126

7. Brnic Z, Hebrang A. 2001. Breast compression and radiation dose in two different mammographic oblique projections: 45 and $60^{\circ}$. European Journal of Radiology 40, 2001:10-15. Elsevier Science Ireland Ltd.

8. NHSBSP 63. Quality Assurance Guidelines for Mammography. April 2006; 42. ISBN 1844630285.

9. Mercer CE, Hogg P, Lawson R, Diffey J, Denton ERE. Practitioner compression force variability in mammography: a preliminary study. Br J Radiol 2013;86:20110596

10. NHSBSP 59. Quality assurance guidelines for breast cancer screening radiology. $2^{\text {nd }}$ edn. March 2011.

11. NHSBSP 33. Quality assurance guidelines for medical physics services. $2^{\text {nd }}$ edn. June 2005; ISBN 1 844630161

12. Report No. 89 (Revision of IPEM Report 59). The commissioning and routine testing of mammographic x-ray systems. Institute of physics and engineering in medicine (IPEM), 2005.

13. D'Orsi CJ, Bassett LW, Berg WA. Mammography, $4^{\text {th }}$ Edition. Breast Imaging Reporting and Data System: ACR BI-RADS ${ }_{\circledast}$. Reston VA: American College of Radiology, 2003.

14. Chida K, Komatsu Y, Sai M, Nakagami A, Yamada T, Yamashita T et al. Reduced compression mammography to reduce breast pain. Clinical Imaging 2009; 33:7-10.

15. Sullivan D.C, Beam C.A, Goodman S.M, Watt D.L. Measurement of force applied during mammography. Radiology 1991; 181: 355-7.

16. Myklebust AM, Seierstad T, Stranden E, Lerdal A. Level of satisfaction during mammography screening in relation to discomfort, service provided, level of pain and breast compression. European Journal of Radiography 2009; 1:66-72. 
17. Pisano E.D, Gatsonis C, Hendrick E. Diagnostic performance of digital versus film mammography for breast-cancer screening. NEngl JMed 2005; 353:1773-83.

18. Oliveira M, Nogueira M.S, Guedes E, Andrade M.C, Peixoto J.E, Joana G.S, et al. Average glandular dose and phantom image quality in mammography. Nuclear Instruments and methods in Physics Research 2007; 580: 574-577. Elsevier B.V.

19. Korf A, Herbst CP, Rae WID. The relationship between compression force, image quality and radiation dose in mammography. SA Journal of Radiology. Dec 2009 